\section{MR Imaging-Based Evidence of Vasogenic Brain Edema in a Case of Acute Acetone Intoxication}

Acute intoxication with acetone is uncommon and presents with neurologic symptoms and high acetone concentrations in brain parenchyma. ${ }^{1}$ Chronic acetone intoxication has been described in painters. $^{2}$

A 31-year-old woman was admitted in a lethargic state with markedly increased muscle tone in the upper extremities. Past medical history included abuse of alcohol and benzodiazepines, borderlinepersonality disorder, and repeated suicide attempts. Fluid-attenuated inversion recovery images (FLAIR; Fig 1) demonstrated well-circumscribed symmetrical hyperintensities with corresponding increased water diffusivity on apparent diffusion coefficient (ADC) maps in the deep gray matter and brain stem. Analysis of serum and urine revealed an extremely high level of acetone $(836 \mathrm{mg} / \mathrm{L} ; 200$-fold above normal) and an elevated level of isopropanol (10 mg/L; 5 -fold above normal). As the serum acetone level decreased in the following days, her clinical symptoms gradually improved. At 1-year follow-up examination, her neurologic status was normal. Accordingly, the MR imaging alterations had resolved.

During fasting, acetone is produced endogenously as a physiologic by-product during the breakdown of fatty acids in the absence of carbohydrates via production of acetoacetic acid and the release of carbon dioxide. This increase of ketone bodies during starvation may lead to a blood acetone concentration of up to $10 \mathrm{mg} / \mathrm{L}$, a finding also seen in diabetic subjects or in intoxication with alcohol or salicylates. ${ }^{3}$ After ingestion of larger amounts of acetone, it is converted into isopropanol, and exogenous isopropanol, in turn, will slowly be metabolized to acetone. The proportion of measured concentrations of these substances in our patient indicates a primary intoxication accompanied by symptoms consistent with previous reports of severe acetone poisoning. ${ }^{4}$ Considering her past medical history, suicidal intention was the most probable cause of its ingestion.

T2-weighted and FLAIR images showed hyperintensities in the putamina, pallidi, and thalami with extension to the brain stem (Fig 1). ADC maps demonstrated increased values indicating vasogenic edema, whereas the transient occurrence of the lesions excluded a necrotic process.

Although none of the previous reports of acute acetone intoxication included MR imaging examinations, lesions affecting the thal- ami, reticular formation of the brain stem, and the basal ganglia in our patient may explain extrapyramidal symptoms and depression of consciousness seen in such patients. Other organic solvents, such as methanol, cause bilateral putaminal cytotoxic edema. The reason why selective putaminal involvement occurs in organic solvent poisoning is not understood but may be due to a relatively higher rate of oxygen and glucose consumption in the striatum. MR imaging findings of methanol intoxication may be explained by the additional cytotoxic effect of formic acid, which accumulates in the striatum and inhibits cytochrome-C oxidase. Toxic metabolites like those seen in methanol intoxication do not form after ingestion of acetone, and, thus, cytotoxic edema is less prominent. Regression of the cerebral lesions, such as those in our patient, seems to indicate that, in acetone intoxication, vasogenic edema plays an important role.

\section{References}

1. Tsatsakis AM, Dolapsakis G, Troulakis G, et al. Fatal and non-fatal outcome by accidental intoxication with paint thinner. J Clin Forensic Med 1997;4:133-37

2. Triebig G, Barocka A, Erbguth F, et al. Neurotoxicity of solvent mixtures in spray painters. II. Neurologic, psychiatric, psychological, and neuroradiologic findings. Int Arch Occup Environ Health 1992;64:361-72

3. Brinkmann B, Fechner G, Karger B, et al. Ketoacidosis and lactic acidosisfrequent causes of death in chronic alcoholics? Int J Legal Med 1998;111:115-19

4. Kostusiak V, Bekkal R, Mateu P. Survival after drinking lethal dose of acetone. Intensive Care Med 2003;29:339

K. Kallenberg

Departments of Neuroradiology and MR-Research in Neurology and Psychiatry

A. Behrens

Department of Pharmacology and Toxicology H. Strik

Department of Neurology M. Knauth

Department of Neuroradiology University Medical Center Göttingen, Germany

DOI 10.3174/ajnr.A0913
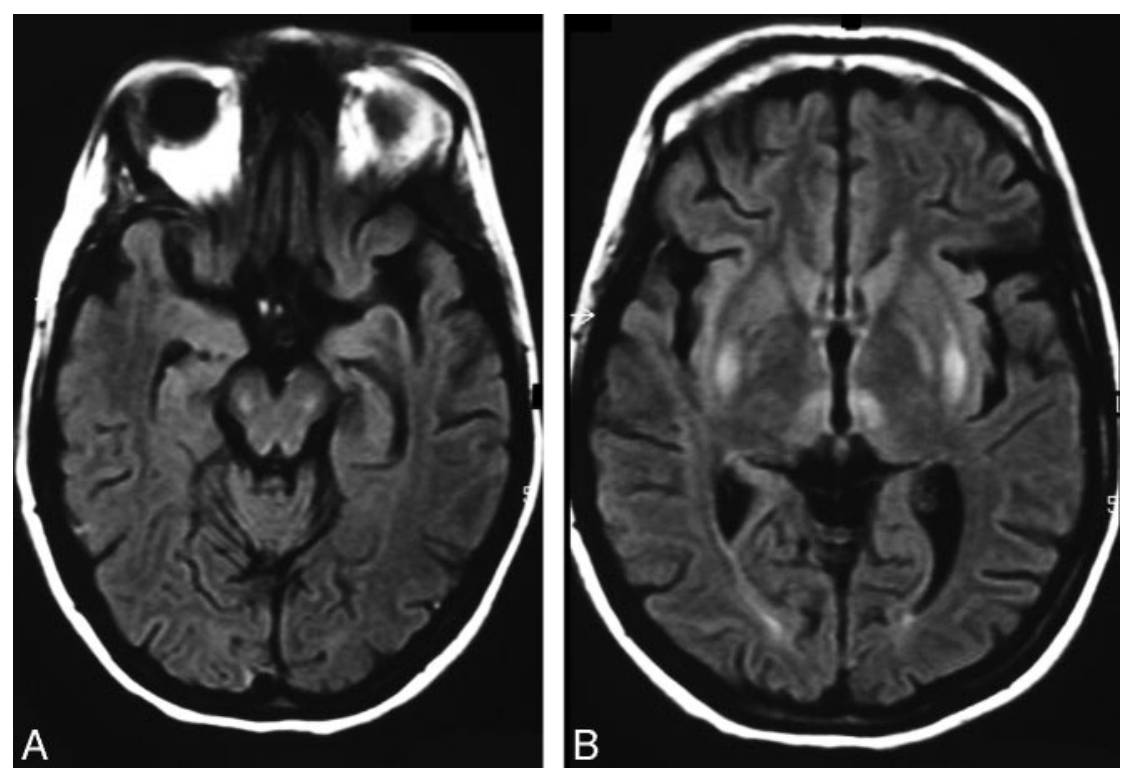

Fig 1. FLAIR image shows bilateral hyperintensities in the putamina, globi pallidi, and thalami (at the level of the nuclei dorsomedialis), area pretectalis, and midbrain (at the lemnisci medialis). 